
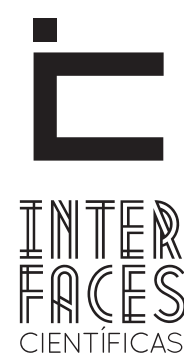

SAÚDE E AMBIENTE

ISSN IMPRESSO 2316-3313

ISSN ELETRÔNICO 2316-3798

\title{
AVALIAÇÃO DA QUALIDADE DE VIDA DE RESIDENTES EM ÁREAS RURAIS
}

Paulo Júnior Paz de Lima
Helenice Bosco de Oliveira²

\section{RESUMO}

A discussão sobre qualidade de vida (QV) no contexto rural tem assumido um aspecto secundário, dada à urgência de outras prioridades. Com isso, objetivou-se avaliar a qualidade de vida de residentes em áreas rurais, em Atibaia/SP e identificar alterações nos domínios do WHOQOL-Bref, quando relacionados a fatores sociodemográficos. 0 estudo foi descritivo entre abril e dezembro de 2011, com 355 participantes, com idade acima de 18 anos. Foram aplicados questionários sociodemográficos e WHOQOL-Bref. Foram realizadas análises descritivas e regressão linear múltipla das variáveis sociodemográficas e domínios do WHOQOL-Bref. Foi realizado ajuste para idade, sexo, estado civil, escolaridade e renda. Foi utilizado o programa SAS, versão 9.2 nas análises. Nível de significância (5\%) e intervalo de confiança (IC) de 95\%. Os resultados apontaram associação estatística entre os domínios do WHOQOL-Bref e as variáveis: idade (físico e social), sexo (físico e psicológico), estado civil (social), escolaridade (psicológico), renda (social e ambiental), tipo de propriedade (ambiental), problema de saúde (físico e psicológico) e uso de medicamento (psicológico e social). Sendo assim, os resultados destacaram a contribuição de variáveis sociodemográficas para melhor QV de residentes em áreas rurais, em um contexto brasileiro. Estes podem servir de parâmetro para estudos futuros com população rural.

\section{PALAVRAS-CHAVE}

Qualidade de vida. População rural. Perfil epidemiológico. 


\section{ABSTRACT}

The discussion on quality of life (QOL) in the rural context has assumed a secondary aspect, given the urgency of other priorities. The aim of this work was to evaluate the quality of life of rural residents in Atibaia/SP, and identify changes in the domains of WHOQOL-Bref, when related to sociodemographic factors. It was performed a descriptive study between April and December 2011, with 355 participants over the age of 18. Sociodemographic and WHOQOL-Bref questionnaires were administered. Descriptive analyzes and multivariate linear regression of sociodemographic variables and WHOQOL-Bref were performed. Adjustment in the variables for age, sex, marital status, education and income was realized. SAS version 9.2 was used for analyzes. The significance level was $5 \%$ and confidence interval of $95 \%$.
The results indicated a statistical association between the domains of WHOQOL-Bref and the variables: age (physical and social), sex (physical and psychological), marital status (social), education (psychological), income (social and environmental), property type (environmental) health problem (physical and psychological), and medication use (psychological and social). Then, the results highlighted the contribution of sociodemographic variables to better QOL of residents in rural areas, in a Brazilian context. These may serve as parameters for future studies of rural population.

\section{KEYWORDS}

Quality of Life. Rural Population. Epidemiological Profile.

\section{RESUMEN}

El debate sobre la calidad de vida (CV) en el contexto rural ha asumido un aspecto secundario, debido a la urgencia de otras prioridades. Con eso, se objetivó evaluar la calidad de vida de los residentes rurales en Atibaia/SP e identificar cambios en WHOQOL-Bref, en relación a factores socios demográficos. Se realizó un estudio descriptivo entre abril y diciembre de 2011, con 355 participantes, mayores de 18 años. Se aplicaron cuestionarios socios demográficos y WHOQOL-Bref. Se realizaron análisis descriptivos y de regresión linear múltiple de las variables socio demográficas y WHOQOL-Bref. Fue realizado ajuste por edad, sexo, estado civil, educación y renta. Se utilizó el programa SAS, versión 9.2 para los análisis. El nivel de significación (5\%) y el intervalo de confianza (IC) de 95\%. Los resultados mostraron

una asociación estadísticamente significativa entre el WHOQOL-Bref y las variables: edad (físicas y sociales), sexo (físicos y psicológicos), estado civil (sociales), educación (psicológicos), renta (social y ambiental), tipo de propiedad (ambiental), problema de salud (física y psicológica) y el uso de medicamentos (psicológica y social). Los resultados ponen de relieve la contribución de los variables socios demográficos a una mejor calidad de vida de los residentes en las zonas rurales, en el contexto brasileño. Éstos pueden servir como punto de referencia para futuros estudios en la población rural.

\section{PALABRAS CLAVE}

Calidad de vida. Población rural. Perfil epidemiológico. 


\section{INTRODUÇ̄̃̃O}

A realidade de grande parte dos residentes em áreas rurais do Brasil estimula a reflexão sobre as condições de vida, de trabalho e de moradia a eles impostas e, consequentemente, sobre o impacto de tais condições em sua qualidade de vida (QV).

Embora o tema qualidade de vida tenha merecido a atenção de pesquisadores em todo o mundo, quando o objeto da investigação é a população rural, a literatura - principalmente a brasileira - silencia. Tais trabalhos têm explorado a QV em pessoas portadoras de enfermidades ou doenças crônicas, transtornos psicoemocionais, portadoras de HIV/Aids, idosos, alcoólatras, trabalhadores urbanos, entre outras (FLECK e cols., 2008).

A discussão sobre as concepções de saúde e QV no contexto e trabalho no campo é importante, porque tem assumido um aspecto secundário, dada à urgência de outras prioridades, bem como a garantia da sobrevivência, mesmo em condições de trabalho degradantes. Neste sentido, Uchoa; Rozemberg; Porto (2002) destacam que nos poucos trabalhos que relacionam QV e trabalho rural, a QV é discutida a partir da articulação entre condições objetivas (estado de saúde, serviços disponíveis, bens e redes sociais de apoio) e o universo de representações objetivas e subjetivas (avaliação do estado de saúde, satisfação com os serviços, recursos materiais e redes sociais de apoio).

\section{QUALIDADE DE VIDA NO CONTEXTO RURAL}

Os estudos sugerem que as comunidades localizadas em áreas dependentes da agricultura, ou com pobreza persistente, têm uma propensão para maiores déficits na QV (MAMMEN; BAUER; LASS, 2009; NZEADIBE; AJAERO, 2010).

Rklaitien; Andrijauskas; Bacevičien (2008) avaliaram a QV em comunidades rurais na Lituânia.
Os resultados sugerem que o estilo de vida e os aspectos sócio-demográficos podem explicar uma parte substancial das diferenças entre a QV de homens e de mulheres. Fatores como menor escolaridade, sedentarismo e obesidade são mais prevalentes entre as mulheres.

No contexto dos Estados Unidos, McGranahan e Beale (2002), sugerem que o declínio econômico e o êxodo rural em massa são fatores a serem considerados na análise da QV. No contexto chinês, segundo Zhang e outros autores (2009), o rápido crescimento econômico aumentou a disparidade de renda entre as zonas urbana e rural a níveis elevados. Com isso, houve drástico impacto na QV dos habitantes das áreas rurais do país, que foram estimulados a migrar para áreas urbanas em busca de uma vida melhor. Essas mudanças demográficas tiveram profundos impactos não apenas no campo, mas também na QV dos migrantes.

No cenário brasileiro, os poucos estudos, também, identificam relações entre a QV no campo, o modo de organização e realização do trabalho (FAKER, 2009; SENA; VARGAS; OLIVEIRA, 2013).

\subsection{OBJETIVOS}

Avaliar a qualidade de vida dos residentes em áreas rurais; identificar alteração dos domínios da QV entre os residentes da área de estudo; identificar alteração em alguns dos domínios da qualidade quando relacionados à função desempenhada no campo e a relação entre sobrecarga de trabalho e alteração dos domínios da QV.

\subsection{MÉTODOS}

Trata-se de um estudo descritivo, com residentes maiores de 18 anos em áreas rurais dos bairros de Laranjal, Pedreira, Ponte Alta e Rio Abaixo, no município de Atibaia (SP). Atibaia possui, segundo o Instituto Brasileiro de Geografia e Estatística - IBGE (BRASIL, 
2010), uma população de 126.603 habitantes (62.211 homens e 64.392 mulheres), assim distribuída: população urbana - 115.229 habitantes ( 56.235 homens e 58.994 mulheres); população rural - 11.374 (5.976 homens e 5.398). Em 2010, Atibaia tinha um Produto Interno Bruto (PIB) de R\$3.062.680,00.

\subsubsection{CÁLCULO AMOSTRAL}

Foi utilizado cálculo de tamanho amostral para estimativa de médias, nos domínios do WHOQOL-Bref, esperado para uma população normal (FLECK et al., 2000). Considerou-se um poder de teste de $80 \%$, com um erro alfa de $5 \%$ e uma possível perda de informação em $20 \%$ dos indivíduos amostrados (ELASHOFF; LEMESHOW, 2006).

\subsubsection{VARIÁVEIS DE ANÁLISES}

Foram consideradas como variáveis dependentes os domínios do WHOQOL-Bref: físico, psicológico, social e ambiental e, como independentes, as variáveis sociodemográficas (sexo, idade, estado civil, cor/ etnia, escolaridade, tipo de moradia e propriedade, renda, ocupação, ser trabalhador rural, se trabalhou na agricultura, carga de trabalho semanal), uso de agrotóxicos, contato com agrotóxicos, intoxicação por agrotóxicos, ingestão de bebida alcoólica, tabagismo, uso de medicamento e problema de saúde.

\subsubsection{INSTRUMENTOS ADOTADOS}

Foram aplicados questionários sócio-demográficos (semiestruturado) e o WHOQOL-Bref, em residentes das áreas de estudo, com idade acima de 18 anos.

O WHOQOL-Bref foi desenvolvido pela Organização Mundial de Saúde (THE WHOQOL GROUP, 1995)e validado para população brasileira por Fleck e outros autores (2000). 0 instrumento consta de 26 questões, sendo duas questões gerais de QV e as demais representativas dos quatro domínios: físico (7 questões), psicológico (6 questões), relação social (3 questões) e meio ambiente (8 questões).

\subsubsection{COLETA DE DADOS}

A coleta de dados ocorreu entre abril e dezembro de 2011. Os participantes foram selecionados por conveniência e de acordo com o interesse dos residentes em participar, voluntariamente, da pesquisa.

Foram aplicados 361 questionarios, sendo a amostra final composta por 355 participantes. Houve uma perda de seis participantes (questionários) que foram excluídos da pesquisa por apresentarem informações incompletas, desistência, duplicidades no preenchimento do questionário pelo mesmo participante e mudança de endereço durante a coleta de dados.

Os critérios de inclusão para participar da pesquisa foram ser maiores de 18 anos e residir nos bairros de estudos no momento da pesquisa. Foram excluídos da pesquisa residentes menores de 18 anos de idade.

Utilizou-se um projeto piloto com 17 participantes das áreas de estudos, com objetivo de testar aplicabilidade dos instrumentos. Os participantes do projeto piloto não foram incluídos nas análises finais do projeto de pesquisa.

As entrevistas foram realizadas por oito (8) agentes e ex-agentes de saúde do Programa de Saúde da Família de Rio Abaixo (PSF Rural de Rio Abaixo), após receberem treinamentos para aplicação dos questionários. As questões eram lidas pelos entrevistadores e anotadas nos questionários, conforme as respostas dadas pelos participantes.

Os entrevistadores informaram sobre a recusa e resistência dos residentes, principalmente dos homens, em participar da pesquisa. Estes justificavam falta de tempo ou de interesse em participar. Embora reiterado sobre a importância de registrar no caderno de campo a recusa e motivo dos residentes em não participar da pesquisa, isto não foi feito pelos entrevistadores. É provável que a maior recusa, por parte dos homens, em participar da pesquisa tenha influen- 
ciado os entrevistadores em maior abordagem/convite às mulheres, o que poderá ter contribuído para maior participação destas.

A informação sobre o número de pessoas que se recusaram a participar da pesquisa fugiu do controle do pesquisador responsável, uma vez que não foram registradas no caderno de campo, conforme esclarecido no treinamento. Diante disso, não foi possível determinar o número de residentes que se recusaram a participar da pesquisa.

\subsubsection{ANÁLISE DOS DADOS}

As informações dos questionários sociodemográficos e dados do WHOQOL-Bref foram digitados em planilhas do programa Excel 2010. Os dados foram analisados a partir do programa SAS, versão 9.2.

Foram realizadas análises descritivas e de regressão linear múltipla das variáveis: sexo, idade, estado civil, cor/etnia, escolaridade, renda, ocupação, ser trabalhador rural, carga de trabalho semanal, tipo de moradia e propriedade, uso de agrotóxicos, contato com agrotóxicos, intoxicação por agrotóxicos, ingestão de bebida alcoólica, tabagismo, uso de medicamento e problema de saúde, além dos domínios do WHOQOL-Bref.

A análise binomial, por sua vez, considerou (sim/ não) para as seguintes variáveis: situação ocupacional, exposições a agrotóxicos, intoxicação por agrotóxco consumo de bebidas alcoólicas, tabagismo e uso de medicamentos. Tais fatores foram correlacionados aos domínios físico, psicológico, social e ambiental, do WHOQOL-Bref.

Realizou-se a análise de associação quanto ao tempo na função, condições relacionadas ao trabalho - jornada de trabalho (número de horas trabalhadas semanalmente) com os domínios do WHOQOL-Bref - e ajustado para as variáveis de idade, sexo, estado civil, escolaridade e renda.
Para identificar variáveis que influenciam na QV, empregou-se a análise de regressão linear múltipla. Devido à ausência de normalidade, foi aplicada a transformação por postos nas variáveis dependentes. O modelo stepwise forward serviu como critério para a seleção das variáveis. 0 nível de significância adotado para os testes estatísticos foi de $5 \%$, com intervalo de confiança (IC) de $95 \%$.

\subsection{6 ÉTICA NA PESQUISA}

O projeto foi aprovado pelo comitê de Ética em Pesquisa da Faculdade de Ciências Médicas da Universidade Estadual de Campinas (FCM/UNICAMP) sob o parecer CEP 1154/2010. A participação dos entrevistados foi voluntária. Os participantes assinaram o Termo de Consentimento Livre e Esclarecido (TCLE) e receberam um Termo de Responsabilidade do Pesquisador. Todos foram informados dos objetivos da pesquisa e sobre a possibilidade de desistência em qualquer etapa do trabalho, sem prejuízo para os desistentes.

\section{RESULTADOS}

A idade média dos participantes é 38,3 anos (DP=14,9), com $61,7 \%$ entre 18 e 40 anos de idade. A maioria é do sexo feminino (64,5\%), de cor/etnia branca (57,5\%), $70,4 \%$ são casados/amasiados e $38 \%$ residem em propriedade própria (Tabela 1 ).

A escolaridade média é de 5,8 anos de estudos $(\mathrm{DP}=3,6)$. A maioria $(55,8 \%)$ não chegou a concluir o ensino fundamental, $8,7 \%$ concluíram e $20 \%$ têm mais de 11 anos de estudos; $23,4 \%$ não possuem renda, $64,2 \%$ recebem até dois salários mínimos (SM). Dentre os entrevistados, $31 \%$ desempenham atividades agrícolas, $72,2 \%$ relataram já ter trabalhado na agricultura, $33,5 \%$ estão desempregados e $66,5 \%$ estão empregados com ou sem registro em carteira profissional (Tabela 1). A média de tempo de trabalho na função atual é 12,7 anos ( $D P=12,2)$ e a média de horas de trabalho semanal são 40,6 horas ( $D P=13,8)$. 
A prevalência de tabagismo e alcoolismo entre os participantes foi de $20,8 \%$ e $19,5 \%$, respectivamente; $40,1 \%$ mencionaram ter problemas de saúde e 40,9\% fazem uso de algum tipo de medicamento (Tabela 1).

Tabela 1 - Características demográficas, socioeconômicas, condições de trabalho, alcoolismo, tabagismo, problemas de saúde e uso de medicamento entre residentes dos bairros: Laranjal, Pedreira, Ponte Alta e Rio Abaixo, em Atibaia/SP - Brasil, 2011

\begin{tabular}{|c|c|c|c|c|}
\hline \multirow{2}{*}{ VARIÁVEIS } & \multicolumn{2}{|l|}{ SEXO } & \multirow{2}{*}{$\mathbf{N}$} & \multirow{2}{*}{$\%$} \\
\hline & Masculino $\mathrm{n}(\%)$ & Feminino $\mathrm{n}(\%)$ & & \\
\hline Sexo & $126(35,5)$ & $229(64,5)$ & 355 & 100,0 \\
\hline \multicolumn{5}{|l|}{ Idade (anos) } \\
\hline $18-30$ & $44(12,4)$ & $84(23,7)$ & 128 & 36,1 \\
\hline $31-40$ & $30(8,5)$ & $61(17,1)$ & 91 & 25,6 \\
\hline $41-50$ & $18(5,1)$ & $39(11,0)$ & 57 & 16,1 \\
\hline $51-60$ & $17(4,8)$ & $32(9,0)$ & 49 & 13,8 \\
\hline$\geq 61$ & $17(4,8)$ & $13(3,6)$ & 30 & 8,4 \\
\hline \multicolumn{5}{|l|}{ Cor/Etnia } \\
\hline Branca & $74(20,9)$ & $130(36,6)$ & 204 & 57,5 \\
\hline Preta/Parda & $49(13,8)$ & $90(25,3)$ & 139 & 39,1 \\
\hline Amarela & $3(0,9)$ & $9(2,5)$ & 12 & 3,4 \\
\hline \multicolumn{5}{|l|}{ Estado Civil } \\
\hline Casado/amasiado & $81(22,9)$ & $168(47,5)$ & 249 & 70,4 \\
\hline Solteiro & $36(10,1)$ & $36(10,2)$ & 72 & 20,3 \\
\hline Separado/divorciado/viúvo & $9(2,5)$ & $24(6,8)$ & 33 & 9,3 \\
\hline \multicolumn{5}{|c|}{ Escolaridade (anos concluídos) } \\
\hline Não estudou & $11(3,1)$ & $17(4,8)$ & 28 & 7,9 \\
\hline $1-4$ & $48(13,5)$ & $89(25,1)$ & 137 & 38,6 \\
\hline $5-8$ & $33(9,3)$ & $59(16,6)$ & 92 & 25,9 \\
\hline $9-10$ & $8(2,3)$ & $19(5,3)$ & 27 & 7,6 \\
\hline$\geq 11$ & $26(7,3)$ & $45(12,7)$ & 71 & 20,0 \\
\hline \multicolumn{5}{|l|}{ Tipo de propriedade } \\
\hline Alugada/Arrendatário & $28(7,9)$ & $58(16,3)$ & 86 & 24,2 \\
\hline Cedida & $46(13,0)$ & $88(24,8)$ & 134 & 37,8 \\
\hline Própria & $52(14,6)$ & $83(23,4)$ & 135 & 38,0 \\
\hline \multicolumn{5}{|l|}{ Renda mensal (em R\$) } \\
\hline Sem renda & $11(3,1)$ & $72(20,3)$ & 83 & 23,4 \\
\hline
\end{tabular}




$$
\begin{aligned}
& \leq 1 \mathrm{SM}^{*} \\
& >1 \leq 2 \mathrm{SM} \\
& >2 \leq 3 \mathrm{SM}
\end{aligned}
$$$$
>3 \leq 5,5 \mathrm{SM}
$$

Ocupação

Desempregado

Empregado com registro

Empregado sem registro

Trabalhador rural

Trabalhou na agricultura

Usa agrotóxico no processo de trabalho

Tem contato com agrotóxico

Sofreu intoxicação por agrotóxico

Sentiu-se mal durante aplicação de agrotóxico

Alcoolismo

Tabagismo

Refere ter problema de saúde

Refere uso de medicamento

SM - Salário mínimo (R\$ 545,00, em 2011).
Fonte: Dados da pesquisa, 2013.

\begin{tabular}{rrrr}
$19(5,4)$ & $73(20,5)$ & 92 & 25,9 \\
$59(16,6)$ & $77(21,7)$ & 136 & 38,3 \\
$26(7,3)$ & $7(2,0)$ & 33 & 9,3 \\
$11(3,1)$ & $0(0,0)$ & 11 & 3,1 \\
& & & \\
$20(5,6)$ & $99(27,9)$ & 119 & 33,5 \\
$72(20,3)$ & $76(21,4)$ & 148 & 41,7 \\
$34(9,6)$ & $54(15,2)$ & 88 & 24,8 \\
$44(13,0)$ & $64(18,0)$ & 108 & 31,0 \\
$93(26,2)$ & $162(46,0)$ & 255 & 72,2 \\
$23(6,5)$ & $25(7,5)$ & 48 & 14,0 \\
$28(8,1)$ & $39(11,0)$ & 67 & 19,1 \\
$20(6,1)$ & $24(7,2)$ & 44 & 13,3 \\
$20(6,2)$ & $33(10,4)$ & 53 & 16,6 \\
$39(11,2)$ & $29(8,3)$ & 68 & 19,5 \\
$31(9,0)$ & $41(11,8)$ & 72 & 20,8 \\
$50(14,1)$ & $92(26,0)$ & 142 & 40,1 \\
$31(8,8)$ & $112(32,1)$ & 143 & 40,9 \\
\hline
\end{tabular}

As médias de respostas nos domínios físico $(72,7)$, psicológico $(71,4)$ e social $(73,3)$ do WHOQOL-Bref apresentaram melhores aspectos, enquanto o ambiental apresentou uma média de 56,9 (Tabela 2).

Tabela 2 - Análise descritiva dos domínios do WHOQOL-Bref entre os residentes rurais dos bairros: Laranjal, Pedreira, Ponte Alta e Rio Abaixo, em Atibaia/SP - Brasil, 2011

\begin{tabular}{lllllll}
\hline WHOQOL-BREF & N & Média & Mínimo & Mediana & Máximo & Desvio Padrão \\
\hline Domínios & & & & & & \\
Físico & 350 & 72,7 & 28,6 & 75,0 & 100,0 & 16,2 \\
Psicológico & 342 & 71,4 & 29,2 & 75,0 & 100,0 & 14,1 \\
Social & 344 & 73,3 & 8,3 & 75,0 & 100,0 & 15,8 \\
Ambiental & 345 & 56,9 & 9,4 & 56,3 & 100,0 & 15,4 \\
\hline
\end{tabular}

Fonte: Dados da pesquisa, 2013.

No modelo de regressão (Tabela 3), os resultados apontaram associações estatísticas das variáveis: idade $(p<0,001)$, sexo $(p<0,001)$ e queixas de problemas de saúde $(p<0,001)$ com domínio físico de qualidade de vida. Em relação ao domínio psicológico, verificou-se associação com sexo $(p<0,003)$, escolaridade $(p<0,006)$ e queixas de problemas de saúde $(p<0,026)$. O domínio social apontou associação com idade 
$(p<0,002)$, estado civil $(p<0,005)$ e renda $(p<0,003)$. Quanto ao domínio ambiental, a associação foi entre renda $(p<0,001)$ e ter residência própria $(p<0,001)$ em relação à alugada e arrendada.

Tabela 3 - Regressão linear múltipla: variáveis que influenciam na qualidade de vida avaliada pelo WHOQOL-Bref

\begin{tabular}{|c|c|c|c|c|c|c|c|c|c|c|c|c|c|c|c|c|}
\hline \multirow{3}{*}{ VARIAVEIS } & \multicolumn{16}{|c|}{ WHOQOL-BREF } \\
\hline & \multicolumn{4}{|c|}{ Domínio Físico } & \multicolumn{4}{|c|}{ Domínio Psicológico } & \multicolumn{3}{|c|}{ Domínio Social } & \multicolumn{5}{|c|}{ Domínio Ambiental } \\
\hline & & $P$ & $R 21$ & $R 22$ & & $P$ & $R 21$ & $R 22$ & & $P$ & $R 21$ & $R 22$ & & $P$ & $R 21$ & $R 22$ \\
\hline Idade & $-1,50$ & 0,0001 & 0,03 & 0,25 & - & - & - & - & $-1,16$ & 0,0025 & 0,04 & 0,12 & - & - & - & - \\
\hline $\begin{array}{l}\text { Sexo: masculi- } \\
\text { no x feminino }\end{array}$ & 42,28 & 0,0001 & 0,04 & 0,25 & 33,64 & 0,0030 & 0,02 & 0,17 & - & - & - & - & - & - & - & - \\
\hline $\begin{array}{l}\text { Estado Civil: c/ } \\
\text { comp x s/comp }\end{array}$ & - & - & - & - & - & - & - & - & 35,62 & 0,0055 & 0,02 & 0,12 & - & - & - & - \\
\hline $\begin{array}{l}\text { Escolaridade: } \\
\text { anos concluídos }\end{array}$ & - & - & - & - & 5,25 & 0,0006 & 0,05 & 0,17 & - & - & - & - & - & - & - & - \\
\hline $\begin{array}{l}\text { Renda (salário } \\
\text { mínimo) }\end{array}$ & - & - & - & - & - & - & - & - & 19,57 & 0,0032 & 0,03 & 0,12 & 25,77 & 0,0001 & 0,06 & 0.10 \\
\hline Propriedade & - & - & - & - & - & - & - & - & - & - & - & - & - & - & 0,04 & 0.10 \\
\hline $\begin{array}{l}\text { Própria X aluga- } \\
\text { da/arrendada }\end{array}$ & - & - & - & - & - & - & - & - & - & - & - & - & 49,55 & 0,0006 & - & - \\
\hline $\begin{array}{l}\text { Cedida X aluga- } \\
\text { da/arrendada }\end{array}$ & - & - & - & - & - & - & & - & - & - & - & - & 25,50 & 0,0802 & - & - \\
\hline $\begin{array}{l}\text { Problema de } \\
\text { saúde (sim x } \\
\text { não) }\end{array}$ & $-68,57$ & $<0,0001$ & 0,18 & 0,25 & $-27,84$ & 0,0268 & 0,01 & 0,17 & - & - & - & - & - & - & - & - \\
\hline $\begin{array}{l}\text { Uso medica- } \\
\text { mento (sim x } \\
\text { não) }\end{array}$ & - & - & - & - & $-32,79$ & 0,0101 & 0,09 & 0,17 & 32,57 & 0,0057 & 0,03 & 0,12 & - & - & - & - \\
\hline
\end{tabular}

$\mathrm{R}^{2} 1=\mathrm{R}^{2}$ parcial - proporção da variabilidade da resposta explicada exclusivamente pelo preditor em questão.

$R^{2} 2=R^{2}$ modelo - proporção de explicação da variável dependente pela variação das variáveis independentes que ficaram no modelo.
No modelo de regressão linear múltipla, quando analisado por sexo (Tabela 4), os resultados mostraram que quanto menor a idade melhor a QV, referentes aos domínios físico (masculino) e social (feminino). Em relação ao estado civil: com companheiro (c/ comp) e 
sem companheiro ( $\mathrm{s} / \mathrm{comp}$ ), também, houve associação $(p<0,019)$. Ter companheira indicou melhor QV no domínio social (masculino). Maior nível de escolaridade apontou associação com melhor QV em relação aos domínios psicológico (feminino) e social (masculino).

Maior renda apontou melhor QV referente ao domínio ambiental (masculino). Não ter trabalhado na agricultura apresentou associação com melhor
QV no domínio psicológico (masculino). Ter casa própria mostrou associação com melhor QV quanto aos domínios ambiental (feminino) e residir em casa cedida no psicológico (masculino). Não ter problema de saúde indicou melhor QV nos domínios físico e psicológico entre os homens e físico entre mulheres. Não fazer uso de medicamento mostrou melhor QV nos domínios psicológico, social e ambiental entre as mulheres.

Tabela 4 - Regressão linear múltipla de variáveis que influenciam nos aspectos da qualidade de vida avaliada pelo WHOQOL-Bref, por sexo

\begin{tabular}{|c|c|c|c|c|c|c|c|c|c|c|c|c|c|c|c|c|}
\hline \multirow{3}{*}{ VARIAVEIS } & \multicolumn{16}{|c|}{ WHOQOL-BREF } \\
\hline & \multicolumn{4}{|c|}{ Domínio Físico } & \multicolumn{4}{|c|}{ Domínio Psicológico } & \multicolumn{3}{|c|}{ Domínio Social } & \multicolumn{5}{|c|}{ Domínio Ambiental } \\
\hline & & $P$ & $R 21$ & $R 22$ & & $P$ & $R 21$ & $R 22$ & & $P$ & $R 21$ & $R 22$ & & $p$ & $R 21$ & $R 22$ \\
\hline \multicolumn{17}{|l|}{ Sexo Feminino } \\
\hline Idade & - & - & - & - & - & - & - & - & $-1,01$ & 0,0038 & 0,05 & 0,08 & - & - & - & - \\
\hline $\begin{array}{l}\text { Escolaridade (anos } \\
\text { concluídos) }\end{array}$ & - & - & - & - & 3,62 & 0,0045 & 0,05 & 0,11 & - & - & - & - & - & - & - & - \\
\hline Propriedade & - & - & - & - & - & - & - & - & - & - & - & - & - & - & 0,07 & 0,10 \\
\hline $\begin{array}{l}\text { Própria X alugada/ } \\
\text { arrendada }\end{array}$ & - & - & - & - & - & - & - & - & - & - & - & - & 42,17 & 0,0003 & - & - \\
\hline $\begin{array}{l}\text { Cedida X alugada/ } \\
\text { arrendada }\end{array}$ & - & - & - & - & - & - & - & - & - & - & - & - & 18,15 & 0,1232 & - & - \\
\hline $\begin{array}{l}\text { Problema de saúde } \\
\text { (sim x não) }\end{array}$ & $-52,22$ & $<0,0001$ & & 0,16 & - & - & - & - & - & - & - & - & - & - & - & - \\
\hline $\begin{array}{l}\text { Uso medicamento } \\
\text { (sim x não) }\end{array}$ & - & - & - & - & $-28,22$ & 0,0021 & 0,06 & 0,11 & $-19,50$ & 0,0389 & 0,03 & 0,08 & $-21,03$ & 0,0232 & 0,03 & 0,10 \\
\hline \multicolumn{17}{|l|}{ Sexo Masculino } \\
\hline Idade & $-0,70$ & 0,0004 & 0,10 & 0,38 & - & - & - & - & - & - & - & - & - & - & - & - \\
\hline $\begin{array}{l}\text { Escolaridade (anos } \\
\text { concluídos) }\end{array}$ & - & - & - & - & - & - & - & - & 1,79 & 0,0459 & 0,03 & 0,15 & - & - & - & - \\
\hline $\begin{array}{l}\text { Estado Civil: c/ } \\
\text { comp x s/comp }\end{array}$ & - & - & - & - & - & - & - & - & 15,85 & 0,0199 & 0,04 & 0,15 & - & - & - & - \\
\hline $\begin{array}{l}\text { Cor/Etnia: Branca x } \\
\text { Não Branca }\end{array}$ & 11,93 & 0,0468 & 0,03 & 0,38 & - & - & - & - & - & - & - & - & - & - & - & - \\
\hline
\end{tabular}


Renda (salário

mínimo)

Trabalhou agricul-

tura (sim x não)

Carga horária de

semanal

Propriedade

Própria X alugada/

arrendada

Cedida X alugada/

arrendada

Problema de saúde

(sim x não

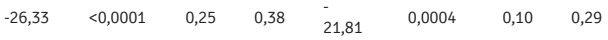

Fonte: Dados da pesquisa, 2013.

$R^{2} 1=R^{2}$ parcial - proporção da variabilidade da resposta explicada exclusivamente pelo preditor em questão.

$R^{2} 2=R^{2}$ modelo - proporção de explicação da variável dependente pela variação das variáveis independentes que ficaram no modelo.

\section{DISCUSSÃO}

A população de estudo é relativamente jovem, $61,7 \%$ com idade até 40 anos. A maioria é do sexo feminino, casados/amasiados e branca. 0 trabalho doméstico feminino e/ou a proximidade das lavouras em relação às residências podem ter contribuído para maior participação das mulheres na pesquisa. Foi mais comum encontrá-las em casa. A maior participação das mulheres, neste trabalho, se deu, também, pela maior recusa por parte dos homens em participar da pesquisa sob a justificativa de falta de tempo ou de interesse. É provável que os entrevistadores tenham convidado mais as mulheres do que os homens, por prever sua recusa. Essa última informação não pôde ser confirmada devido à falta de registro sobre recusa no caderno de campo, embora reiterada sua importância.

Os residentes rurais, em específico os trabalhadores rurais, geralmente temem participar de pesquisas e dar informações sobre o processo de trabalho. Eles desconfiam que as pesquisas estejam relacionadas à investigação sobre emprego de praguicidas na lavou- ra, visto que muitos desses produtos, embora proibidos em seus países de origem, ainda são empregados no Brasil. Nos estudos de Fernández e outros autores (2006) e Lima (2009) também se verifica uma maior participação de mulheres. Lima (2009) destaca que a baixa procura dos homens aos serviços de saúde para expor seus problemas é, também, um fator responsável pela recusa em participar de pesquisas.

A renda mensal dos participantes é de até dois salários mínimos, com média salarial de R\$ 619,90, considerados como pessoas de baixa renda. Dados do IBGE (BRASIL, 2010a) destacam 32,7\% de pessoas ocupadas no Brasil, em 2010, com rendimento médio de até dois salários mínimos.

A maioria dos participantes (63,7\%), incluindo os que não estudaram, não chegou a concluir o ensino fundamental. Segundo o IBGE (BRASIL, 2010a), houve um aumento no nível de instrução da população brasileira entre 2000 e 2010. O percentual de pessoas com mais de 10 anos de idade sem instrução ou com ensino fundamental incompleto caiu de $65,1 \%$ para $50,2 \%$. 
Estudos apontam as dificuldades econômicas e a falta de exigência de escolaridade para o trabalho rural como responsáveis pelo fato dos homens abandonarem a escola muito cedo, no intuito de reforçar o sustento da família (LIMA, 2009). 0 emprego de novas tecnologias para produção e desenvolvimento da agricultura (voltada para a exportação de alimentos, principalmente) têm exigido melhor qualificação e maior escolaridade do homem do campo (PERES, 2009).

Investimentos em educação podem proporcionar melhores oportunidades de emprego e de rendas mais elevadas. Melhores níveis de educação geralmente são associados a maiores níveis de renda e melhor satisfação com a vida. Famílias de baixa renda e famílias rurais são, especialmente, vulneráveis à pobreza, mudanças de emprego e menor renda, doenças e mudanças de estado civil. É mais provável encontrar taxas elevadas de pobreza persistente nas comunidades rurais (MAMMEN; BAUER; LASS, 2009).

As médias dos domínios do WHOQOL-Bref foram melhores em relação aos aspectos físico, psicológico e social. A exceção foi o ambiental $(56,9)$, que indicou pior desempenho, quando comparados com outros estudos. Lima (2009), também, identificou o domínio ambiental como fator de pior aspecto na QV, entre trabalhadores rurais de Três Pontas/MG, em 2005.

Os resultados apontaram as variáveis: idade, sexo, estado civil, escolaridade, renda, tipo de posse de propriedade, queixas de problemas de saúde e uso de medicamento como associadas estatisticamente aos domínios de qualidade de vida. Na estratificação por sexo, além das variáveis já relacionadas, cor/etnia branca, não ter trabalhado na agricultura e maior carga horária de trabalho, também, mostraram associação com melhor qualidade de vida entre os homens, principalmente, em relação aos domínios físico, psicológico e social, respectivamente.

Fatores como idade avançada, baixa escolaridade, assim como sofrimento mental, problemas de saúde e efeito colateral de medicamento contribuem para um baixo nível de QV. Tais variáveis podem contribuir negativamente para autoavaliação de saúde e afetar diferencialmente domínios de QV devido a fatores específicos da doença (JAKOBSSON; HALLBERG; WESTERGREN, 2004; PATRICK et al., 2004). Pereira e outros autores (2011) destacaram a associação entre sexo feminino e baixos escores no domínio ambiental e, ainda, o uso de medicamento como variáveis associadas a baixos escores de QV no domínio psicológico. Jakobsson; Hallberg; Westergren (2004) identificaram idade, problema de saúde, sexo feminino, renda e falta de companheiro associados à baixa QV.

Embora o estudo tenha sido realizado com residentes de áreas rurais, apenas $31 \%$ dos residentes são trabalhadores rurais. A maioria desempenha atividades laborais em áreas não agrícolas. A maioria, 72,2\% mencionou ter trabalhado na agricultura e $36,3 \%$ relataram ter trabalhado no campo antes da função atual.

Em relação ao contato e exposição a agrotóxico, seja no processo de trabalho ou por residir próximos as lavouras, $18,9 \%$ relataram ter contato. Quando questionados sobre como ocorre o contato, $25,2 \%$ mencionaram morar próximo às plantações, sentir o cheiro durante a aplicação de agrotóxicos, puxar a mangueira para aplicação de agrotóxico por outro, permanecer próximo quando os agrotóxicos são aplicados e lavar roupas usadas durante a aplicação desses produtos. Em relação à intoxicação por agrotóxico, 13,3\% mencionaram ter sofrido intoxicação. No entanto, o número dos que relataram ter se sentido mal durante a aplicação desse tipo de defensivo foi maior, $16,6 \%$. Os estudos de Sena; Vargas; Oliveira (2013) com trabalhadores rurais de Lagarto, em Sergipe, apontaram piores escores de QV entre os agricultores que utilizavam agrótixicos no processo de trabalho, quando comparados aos que não utilizavam.

A inclusão neste estudo, de variáveis referentes à exposição e intoxicação por agrotóxicos tinha como 
finalidade verificar a associações entre essas variáveis e domínios de qualidade de vida do WHOQOL-Bref. Por se tratar de um estudo com residentes em comunidades rurais, esperava-se encontrar relação entre QV e variáveis como uso de agrotóxico no processo de trabalho, contato com agrotóxico, intoxicação por agrotóxico. No entanto, esta hipótese não foi confirmada, embora estudos apontem uma taxa média de letalidade de $0,4 \%$ e mortalidade por agrotóxicos de 3,4\%, no Brasil, em 2001, além de altas taxas de intoxicações (PIRES; CALDAS; RECENA, 2005; FARIA; FASSA; FACCHINI, 2007).

Entre a população rural estudada, a prevalência de tabagismo foi de $20,8 \%$ e de alcoolismo $19,5 \%$. Esses números podem estar subestimados. Esses dados sobre a "negação" de hábitos foram contestados, no momento da entrevista, por pessoas próximas que informaram sobre os hábitos de fumar e de ingerir bebida alcoólica dos entrevistados. A prevalência de tabagismo entre os Estados brasileiros e Distrito Federal, em 2008, foi de $16,1 \%$. A frequência de adultos tabagistas variou entre $9,7 \%$ e $20,9 \%$ entre as capitais (MALTA et al., 2010).

Em relação ao alcoolismo, Laranjeira e outros autores (2007) apontaram que $52 \%$ dos brasileiros acima de 18 anos de idade podem ser classificados como bebedores e $12 \%$ da população tem algum problema com o álcool. Na população rural, do Vale do Taquari (RS), em 2005, Souza e outros autores (2011) verificaram consumo de álcool em $54,4 \%$ dos participantes.

Outros achados como morbidade e uso de medicamento também são importantes para entender QV no contexto rural. A taxa de morbidade é relativamente alta na população investigada, pois $40,1 \%$ dos entrevistados mencionaram ter algum problema de saúde e $40,9 \%$ fazem uso de algum tipo de medicamento. Pinheiro e outros autores (2002) informam que, segundo dados da Pesquisa Nacional por Amostra de Domicílios (PNAD), de 1998, 23\% da população autoavalia seu estado de saúde como deficiente, sendo que $31,6 \%$ apresentaram, pelo menos, uma doença crônica e 6,9\% referem restrição de atividades por motivo de saúde. As mulheres apresentam maior percentual de restrição de atividades em relação aos homens. As diferenças entre gêneros estreitam-se a partir dos 65 anos nas áreas urbanas e rurais, mas variam com a situação de moradia urbana ou rural.

\section{CONCLUSÃO}

Neste estudo, o reduzido diálogo com estudos nacionais se deve à limitação de pesquisa sobre QV no contexto rural brasileiro, havendo necessidade de assumir outros estudos como referências.

Os resultados apontaram associação estatisticamente significativa entre variáveis sociodemográficas (idade, sexo, estado civil, escolaridade, renda, tipo de posse de propriedade), problemas de saúde e uso de medicamento e os domínios de qualidade de vida. Cor/ etnia branca, não ter trabalhado na agricultura e maior carga horária de trabalho, mostraram associação com melhor qualidade de vida entre os homens.

Os resultados destacaram a contribuição de variáveis sociodemográficas para melhor QV de residentes em áreas rurais, em um contexto brasileiro Estes podem servir de parâmetro para estudos futuros com população do campo.

\section{REFERÊNCIAS}

BRASIL. Instituto Brasileiro de Geografia e Estatística - IBGE (2010). Cidades. Disponível em: <http://www. ibge.gov.br/cidadesat/link.php?uf=sp>. Acesso em: 22 mar. 2013.

BRASIL. Instituto Brasileiro de Geografia e Estatística (IBGE). Censo demográfico 2010. Resultados gerais da amostra. Rio de Janeiro: IBGE, 2010a. 
ELASHOFF, J. D.; LEMESHOW, S. Sample size determination in epidemiologic studies. In: AHRENS, W.; PIGEOT, I. Handbook of epidemiology. New York: Springer, 2006.

FAKER, J. N. A cana nossa de cada dia: saúde mental e qualidade de vida em trabalhadores rurais de uma usina de álcool e açúcar de Mato Grosso do Sul. 2009. 189 f. Dissertação (Mestrado em Psicologia) - Universidade Católica Dom Bosco, Campo Grande (MS), 2009.

FARIA, N. M. X.; FASSA, A. G.; FACCHINI, L. A. Intoxicação por agrotóxicos no Brasil: os sistemas oficiais de informação e desafios para realização de estudos epidemiológicos. Ciência e Saúde Coletiva, v.12, n.1, 2007, p. 25-38.

FERNÁNDEZ, C. F. et al. La depresión en el anciano en una zona rural y su interacción con otros factores. Actas Espanõlas de Psiquiatría, v.34, n.6, 2006, p. 355-361.

FLECK, M. P. A. e cols. Avaliação de qualidade de vida: guia para profissional da saúde. Porto Alegre: Artemed, 2008.

FLECK, M. P. A. et al. Aplicação da versão em português do instrumento abreviado de avaliação da qualidade de vida “WHOQOL-Bref”. Rev Saúde Pública, v.34, n.2, 2000, p. 178-183.

JAKOBSSON, U.; HALLBERG, I. R.; WESTERGREN, A. Overall and health related quality of life among the oldest old in pain. Qual Life Res, v.13, n.1, 2004, p. 125-136.

LARANJEIRA R. et al. levantamento nacional sobre os padrões de consumo de álcool na população brasileira. Brasília: Secretaria Nacional Antidrogas, 2007.

LIMA, J. M. M. Distúrbios do sono, sintomas de ansiedade e de depressão e qualidade de vida em trabalhadores rurais em períodos de entressafra cafeeira. 2009. 94 f. Dissertação (Mestrado em Ciências) - Faculdade de Medicina da Universidade de São Paulo, São Paulo, 2009. MALTA, D. C. et al. Prevalência do tabagismo em adultos residentes nas capitais dos estados e no Distrito Federal, Brasil, 2008. J Bras Pneumol, v.36, n.1, 2010, p. 75-83.

MAMMEN, S.; BAUER, J. W.; LASS, D. Life satisfaction among rural low-income mothers: the influence of health, human, personal, and social capital. Applied Research Quality Life, n.4, 2009, p. 365-386.

MCGRANAHAN, D. A.; BEALE, C. L. Understanding rural population loss. Rural America, v.17, n.4, 2002, p. 2-11.

NZEADIBE, T. C.; AJAERO, C. K. Assessment of socio-economic characteristics and quality of life expectations of rural communities in Enugu State, Nigeria. Applied Research Quality Life, v.5, n.4, 2010, p. 353-371.

PATRICK, J. H. et al. The effects of depressed affect on functional disability among rural older adults. Quality of Life Research, n.13, 2004, p. 959-967.

PEREIRA, R. J. et al. Influência de fatores sociossanitários na qualidade de vida dos idosos de um município do Sudeste do Brasil. Ciência e Saúde Coletiva, v.16, n.6, 2011, p. 2907-2917.

PERES, F. Saúde, trabalho e ambiente no meio rural brasileiro. Ciência e Saúde Coletiva, v.14, n.6, 2009, p. 1995-2004.

PINHEIRO, R. S. et al. Gênero, morbidade, acesso e utilização de serviços de saúde no Brasil. Ciência e Saúde Coletiva, v.7, n.4, 2002, p. 687-707.

PIRES, D. X.; CALDAS, E. D.; RECENA, M. C. P. Uso de agrotóxicos e suicídios no Estado do Mato Grosso do Sul, Brasil. Cad Saúde Pública, v.21, n.2, 2005, p. 598-605.

RKLAITIEN, R.; ANDRIJAUSKAS, K.; BACEVIČIEN, M. Assessment of the quality of life among Lithuanian rural community. Acta Medica Lituanica, v.15, n.3, 2008, p. 156-162. 
SENA, T. R. R.; VARGAS, M. M.; OLIVEIRA, C. C. Saúde auditiva e qualidade de vida em trabalhadores expostos a agrotóxicos. Ciência e Saúde Coletiva, v.18, n.6, 2013, p. 1753-1761.

SOUZA, A. et al. Avaliação do impacto da exposição a agrotóxicos sobre a saúde de população rural. Vale do Taquari (RS, Brasil). Ciência e Saúde Coletiva, v.16, n. 8, 2011, p. 3519-3528.

THE WHOQOL GROUP. The World Health Organization quality of life assessment (WHOQOL): position paper from the World Health Organization. Soc Sci Med, v.41, n.10, 1995, p.1403-1409.
Recebido em: 22 de Dezembro de 2013
UCHOA, E.; ROZEMBERG, B.; PORTO, M. F. S. Entre a fragmentação e a integração: saúde e qualidade de vida de grupos populacionais específicos. IESUS - Informe Epidemiológico do SUS, v.11, n.3, 2002, p.115-128.

ZHANG, J. et al. Discrimination experience and quality of life among rural-to-urban migrants in China: the mediation effect of expectation-reality discrepancy. Qual Life Res, v.18, n.3, 2009, p. 291-300.
1. Doutorando em Saúde Coletiva pela Faculdade de Ciências Médicas da Universidade Estadual de Campinas - UNICAMP e mestre em Saúde Pública pela Faculdade de Saúde Pública da Universidade de São Paulo - FSP/USP. E-mail: pjuniorpl@hotmail.com

2. Docente do doutorado em Saúde Coletica - UNICAMP. E-mail: helenice@ fcm.unicamp.br 